\title{
Hazard Assessment and Risk Analysis in Two Pork Slaughterhouses
}

\author{
Ionut Vlad CORDIŞ ${ }^{1 *}$, Marian MIHAIU ${ }^{1}$, Alexandra TĂBĂRAN ${ }^{1}$, Sorin Daniel DAN ${ }^{1}$, Oana REGET ${ }^{1}$, Darius \\ CORDEA $^{1}$ \\ ${ }^{1}$ Department of Animal Breeding and Food Safety, University of Agricultural Sciences and Veterinary \\ Medicine, Cluj-Napoca, Romania; \\ *Corresponding author:dalv_civ@yahoo.com
}

Bulletin UASVM Veterinary Medicine 72(2) / 2015,

Print ISSN 1843-5270; Electronic ISSN 1843-5378

DOI:10.15835/buasvmcn-vm: 11447

\begin{abstract}
The globalization of the food chain has led nowadays to the increase of the risks related to the dissemination of food poisoning episodes which extend over international borders. Due to this fact, risk analysis of each step of the production chain „from farm to fork" has become necessary. The specific aim of this study was to perform risk analysis in two swine slaughtering units and to highlight the microbiological hazards taking into account the performed analyses. The technological steps that present important risks for meat hygiene state and could jeopardize the production were the refrigeration step and the storage step. In the small capacity slaughterhouse „B”, there was a severe operational deficiency noticed, consisting of the inaccurate removal of the tonsils, which can lead to a high risk of microbial contamination. During the evaluation of the probability for the risk of occuring, in the big capacity slaughterhouse there was a low estimated probability (0-10\%) compared to unit „B” where the probability was high $(>80 \%)$, mainly due to inaccurate removal of the tonsils. During evaluation of risk exposure, with the help of representative scales, marked with colours according to severity, there was a high tolerance noticed in the case of unit „B” represented with the red colour. Following the way of communication and report making, both units presented deficienciesin establishing the alert mechanisms.
\end{abstract}

Keywords: assessment, microbial, risk, slaughterhouse, unit

\section{INTRODUCTION}

Pork meat is one of the most consumed products in Romania. The production of this type of meat in the European Community is increasing and there are amendments (95/23/EEC) on the respective topic that specifically require the application of safety ensuring programs like the HACCP systems. This system is basically a scientific approach to process control, designed to prevent, reduce or eliminate identified hazards in food products (Kukay et al., 1996). The correct and efficient implementation of HACCP systems must be based on accurate data and especially on the assessment of contamination levels in each stage of the production line (Gill et al., 1995). This assessment which is made at the unit will involve the evaluation of the severity and probability of the risks for the product and consumer's safety, taking each hazard individually (Gill and Jones, 1997). Although there are a lot of common points to the HACCP plan applied in pork slaughter processes, each plan must be implemented individually and adapted to the unit. This fact is very important, taking into consideration that slaughter plants differ in terms of range and levels of contamination. Each slaughterhouse needs to have its own baseline data and personalized HACCP plan to match site specific circumstances and processes (Vanne et al., 1996). 
The biological hazards are considered the most dangerous to consumers' health. Previous studies have shown the occurrence of pathogens in pork meat due to contamination during the slaughtering processes. For example, a study made in Ireland found pork products to be contaminated with Listeria (45\%, Sheridan et al., 1994), Yersinia (100\%, Logue et al.,1996), and Salmonella (9.9\%, Duffy et al., 1999). Other statistics show that in Netherlands the prevalence of microbial contamination reaches about 100,000 cases per year, of which a concerning percent of $15 \%$ is associated with pork consumption (Berends, 1998). In many of the food poisoning cases caused by contaminated pork meat, the causative agent was traced back to local pig abattoirs. The fact that pork meat is susceptible to contamination during slaughtering procedures is a major concern because it generates a great economic impact and thus severe financial losses when such outbreaks occur. The Economic Research Service (ERS) of the United States Department of Agriculture (USDA) estimated that food-borne Salmonella infections associated with pork products in the US cost $\$ 0.1$ to 0.2 billion per year (Frenzen et al., 1999).

Because of the great impact mentioned above, our aim was to assess the implementation of the HACCP system in two pork slaughtering units " $\mathrm{A}$ " and "B" which differ in technology and capacity, and to perform a risk analysis so as to evaluate the microbiological hazards and the possible impact on consumer's health.

\section{MATERIALS AND METHODS}

The risk analysis was applied within two pork slaughtering units situated in Sălaj county, different from one another in terms of processing technology and capacity. Slaughterhouse "A" has a large capacity and it is authorized for intracommunity exchanges. Slaughterhouse „B” is a small capacity unit with a limited number of slaughtered animals per day.

Both units are functioning within the sanitaryveterinary legislation regarding the slaughtering activity. The slaughterhouse „A" implemented the HACCP system in 2007, consequently to a Sapard program that led to the modernization of the entire slaughtering line. The unit's advantages are the fact that it can produce a larger amount of carcasses, being able to process approximately 250 tons/week, with each of the spaces being properly designed according to European legislation requirements.

The risk identification was done after a detailed description of the technological process in both analyzed units and taking into consideration the results obtained at the microbiological assessments which are specified in the units' documents, mandatory in the strategic programs. Their evaluation was made taking into account all the requirements stipulated in the HACCP program and risk evaluation manuals.

\section{RESULTS AND DISCUSSION}

In order to evaluate the potential risks for the safety and health of the consumer we created the representative flow diagram for each unit and analyzed each step in both slaughtering plants.

After the analysis performed in slaughterhouse „A" we identified as critical control points for biological hazards the refrigeration and storage steps. This conclusion was drawn taking into account the fact that it might be possible for bacteria to develop if the spaces between the carcasses is not appropriately managed and during our visit the character of non-compliance was evident. The temperature was monitored constantly, but at relatively close intervals, the temperature graphic not being made in time.

At the analysis performed in slaughter plant „B" we identified as critical control points the evisceration, refrigeration and storage steps. The major shortcoming found in unit „B" was the fact that the evisceration and post-mortem inspection was not performed properly. One the major deficiencies found was the fact the tonsils had not been completely removed (Fig.1), some of the carcasses being left with the entire tonsils attached (Fig.2). This poses an important risk for the safety of the product, given the fact that these organs are the entrance gate for bacteria, where the largest amount of germs can be found.

The refrigeration chamber in slaughterhouse „B” is constantly monitored, but the temperature graphic is not made properly and it was obvious that the variations in temperature were relatively high, reaching almost $7^{\circ} \mathrm{C}$ in some cases. This also counts as an important risk, consideringthat an inappropriate temperature will lead to the rapid increase in surface and in depth bacterial counts of the carcasses. 
It was noticed that within the storage areas, the carcasses did not respect the proper distance (Fig. 3 ) which represents a risk, just like in the case of plant „A". The storage conditions in slaughterhouse „B” were not proper and the hygiene conditions demanded improvement.

Attention was paid in particular to the postmortem inspection of the organs and carcasses in both units studied. The post-mortem inspection was done according to the requirements set forth by the EU legislation, the heart was sectioned for cysticercosis examinatio, the lymph nodes inspected properly, all suspicious organs removed and sampled. In case of slaughterhouse „B”, the major deficiency in post-mortem inspection was noticed due to the persistence of the tonsils. The rest

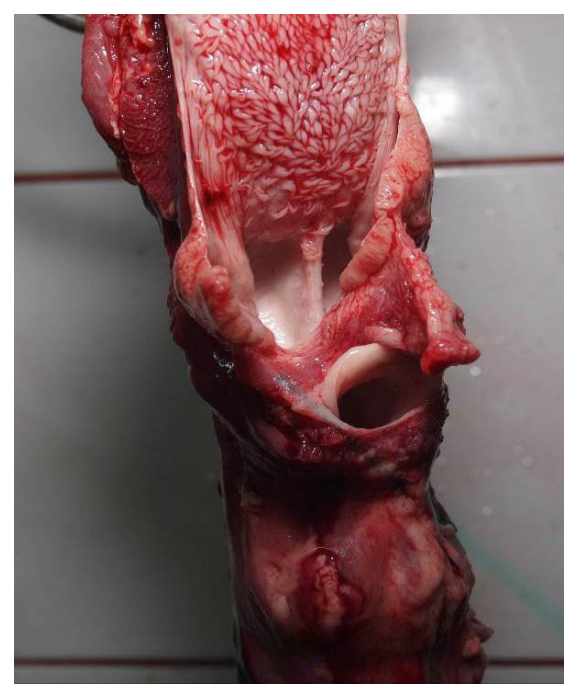

Fig.1. The incomplete removal of the tonsils

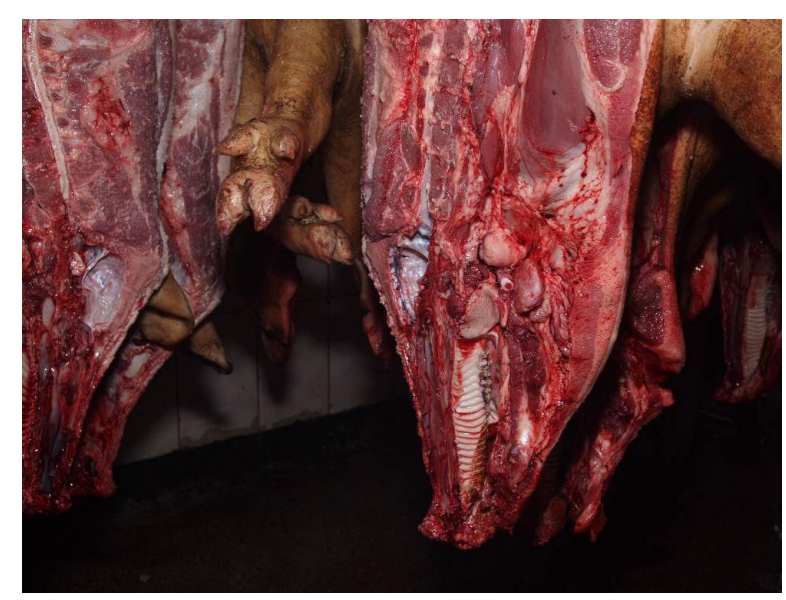

Fig. 2. The lack of tonsils removal and their presence at the carcass of the procedures were performed appropriately, although the system of identification and the correspondence between organs and carcasses was not appropriate.

In order to respect the implementation protocol for the internal control standard, called „risk management”, the subjectivity was reduced by using two complementary methods of identifying the risks described in this methodology. The first was the self-assessment of the risk, which has as main advantage the fact that the one making it is much more familiar with the problems that the unit has. The autoevaluation in the analyzed units has underlined the same risks as we did in our inspection. Acknowledging the existence of these risks, unit „B” is in course of modernization, the spaces improved and the technology devices replaced by more performant ones.

Following the study made in the two slaughtering units, it was revealed that based on the impact and probability scales, the tolerance for the hazards identified is low in some cases. For example, in case of the storage areas, the hazard identified shows a low risk and a high tolerability, given the fact that all results from the documents held by the unit showed no increase in bacterial count. In the case of the refrigeration steps, the tolerability was medium, because the variation of temperature can cause severe effects and the results showed that this step is critical in maintaining the quality of the product. The lowest tolerance index was found at the analysis of the tonsil removal step and this aspect concerned only plant „B” (Tab.1).

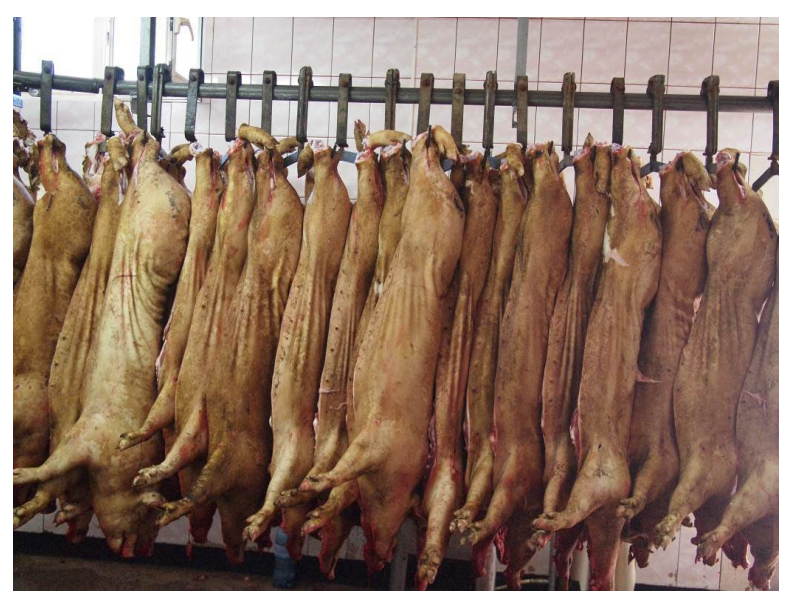

Fig.3. Storage areas of carcasses 
Tab. 1. The evaluation of risk tolerance using qualitative scales and specific colours

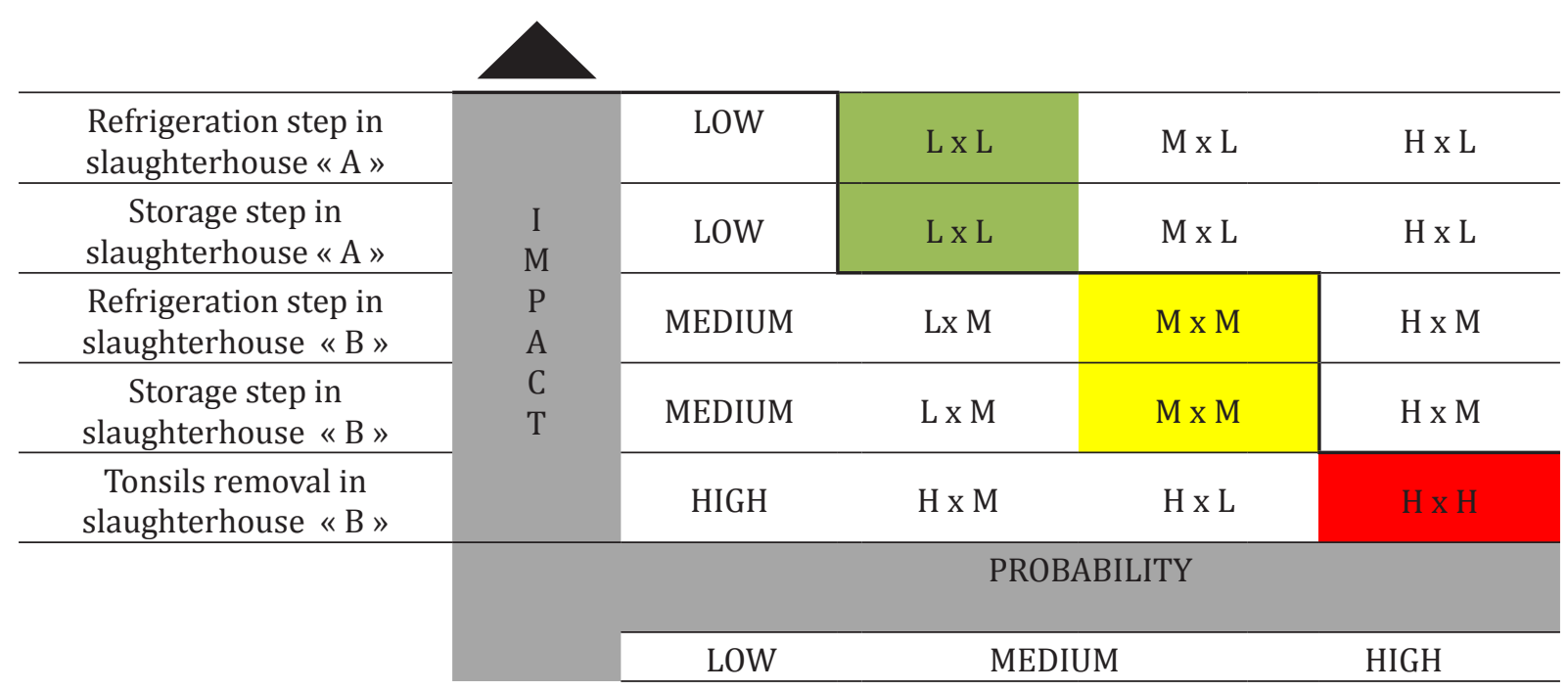

The results obtained following the evaluation of risks which were found to be above the limit of tolerancemustbe considered by adoptingmeasures which will prevent the hazard from occurring. This implies in the case of tonsils removal a new revision of the operation procedures, especially the GMPs (Good Manufacturing Practices) so as to instruct the operators and achieve higher efficiency in this step. Following our investigation it was noticed that according to the tolerance evaluation of the identified risks, the intensity of control measures is directly proportional to the deviation of risk exposure towards the tolerability limit expressed in the beginning of the assessment. For this reason, colours were used in the studied units to visualize the risk profiles. For example, the risks identified as having a higher incidence and that exceed the limits of risk tolerability were highlighted in red, notifying that corrective actions should be taken. The yellow colour marks "attention required", because risks can exceed the tolerability line. The green colour was given for the risks that were situated below the tolerability limit.

The European Comission applies the same risk asssessment system as the one presented in this paper, the only difference being that it uses colour flags according to risk tolerability. For the complex analysis of risk exposure we have made a detailed study from the current literature related to data on human contamination after pork meat consumption. It was found that the highest incidence of microbial contamination in pork meat was with Salmonella spp. (EFSA, 2010).
According to the EFSA report, Salmonella positive samples were found in a relatively high proportion of pig meat, six of 20 reporting countries found none. Data on serovars were incomplete, but $S$. typhimurium was the most common. For the evaluation of disease severity in humans as a result of pathogen transmission by pork meat ingestion we used two numerical indicators: the hospitalization cases and the death rate reported until this hour in the field literature. The quality evaluation of the risk has indicated that Salmonella and L. monocytogenes along with $\mathrm{Cl}$. botulinum and Mycobacterium spp. hold the highest severity. Moreover, the assessment of socio-economical consequences of bacterial diseases, with the involved costs, has revealed that the disease caused by these risks has lead to total costs 10 times higher than in the case of other risks.

\section{CONCLUSION}

In the examined pork slaughterhouses we have identified a series of hazards that can cause serious risks for the safety of the product and consumer health. The most severe hazard identified was the incomplete removal of the tonsils which might lead to the dissemination of dangerous bacteria in the entire carcass. The risk assessment performed within the two units concluded that a revision of the HACCP plan is needed in both of them given the lack of proper monitoring during storage and refrigeration steps which are considered critical control points. 
Acknowledgments: This paper was published under the frame of European Social Found, Human Resources Development Operational Programme 2007-2013, project no. POSDRU/159/1.5/136893.

\section{REFERENCES}

1. Berends BR (1998). A risk assessment approach to the modernization of meat safety assurance. Thesis, Utrecht University, The Netherlands.

2. Duffy G, Cloak OM, O'Sullivan MG, Guillet A, Sheridan JJ, Blair IS, McDowell DA (1999). The incidence and antibiotic resistance profiles of Salmonella spp. on Irish retail meat products. Food Microbiology 16: 623 - 631.

3. Frenzen PD, Buzby JC, Roberts T (1999). An updated estimate of the economic costs of human illness due to foodborne Salmonella in the United States. Proceedings of the 3rd International Symposium on the Epidemiology and Control of Salmonella in Pork, Washington, DC, 215 218.

4. Gill CO, Jones T (1997). Assessment of the hygienic characteristics of a process for dressing pasteurized pig carcasses. Food Microbiology 14:81 - 91.
5. Gill CO, Jones T (1995). The presence of Aeromonas, Listeria and Yersinia in carcass processing equipment at two pig slaughtering plants. Food Microbiology 12:135 141.

6. Kukay CC, Holcomb LH, Sofos JN, Morgan JB, Tatum JD, Clayton PP, Smith GC (1996). Applications of HACCP by small-scale and medium-scale meat processors. Dairy, Food and Environmental Sanitation 16(2):74 - 80.

7. Logue CM, Sheridan JJ, Wauters G, McDowell DA, Blair IS (1996). Yersinia spp. and numbers, with particular reference to Y. enterocolitica bio/serotypes, occurring on Irish meat and meat products, and the influence of alkali treatment on their isolation. International Journal of Food Microbiology 33:257 - 274.

8. Sheridan JJ, Duffy G, McDowell DA, Blair IS (1994). The occurrence and initial numbers of Listeria in Irish meat and fish products and the recovery of injured cells from frozen products. International Journal of Food Microbiology 22:105 - 113.

9. Vanne L, Karwoski M, Karppinen S, Sjoberg AM (1996). HACCP-based quality control and rapid detection methods for micro-organisms. Food Control 7(6):263 - 276. 